\title{
Stability Analysis of a Class of Biological Network Models ${ }^{\dagger}$
}

\author{
Nader Motee ${ }^{1}$, Bassam Bamieh ${ }^{2}$, and Mustafa Khammash ${ }^{2}$
}

\begin{abstract}
In this paper, we establish stability conditions for a special class of interconnected systems arisen in several biochemical applications. It is known that most of the biochemical processes can be represented using quasi-polynomial systems. We show that a special class of quasi-polynomial systems can be cast in the Lotka-Volterra canonical form. We study the asymptotic stability properties of a class of quasipolynomial systems which are relevant to biological network models. First, we show that under some sufficient conditions the solutions of the quasi-polynomial systems (in the positive orthant) converge to the set of equilibrium points. These results are applied to parameterized models of three different biological systems: generalized mass action (GMA) model, an oscillating biochemical network, and a reduced order model with Hill function. We show that one can find the range of parameters for which a given parameterized model is stable.
\end{abstract}

\section{INTRODUCTION}

Biological systems are usually robust against changes in environmental conditions. However, in almost all biological systems, certain parameters are subject to tight control and even small deviations result in malfunction or even death of the system. Examples for such parameters include the operating temperature of the human brain and blood glucose levels. From a system theorist perspective, once we have attained an understanding of network dynamics, a dynamic analysis may yield useful predictions of unknown interactions. A stability analysis can be done using only the network structure, without knowing the rate constants for a particular reaction. The traditional stability analysis and sensitivity analysis, which is based on some knowledge of steady-state behavior, can only provide limited insights into how systems behaves under small perturbations. Therefore, in order to analyze the network behavior under large perturbations, we need to develop an understanding of the system behavior away from equilibrium points.

There is a considerable amount of literature on stability analysis of biochemical network models. In [1], the effect of autocatalysis in a glycolytic pathway model is studied by tools from linear control theory. The authors show that glycolytic oscillations are rather a result of the hard tradeoffs that emerges from the autocatalytic mechanism of glycolysis.

\footnotetext{
1 The author is with the Control and Dynamical Systems Department, California Institute of Technology, 1200 E. California Blvd, Pasadena, CA 91125 USA (motee@cds.caltech.edu).

2 The authors are with the Department of Mechanical Engineering, University of California, Santa Barbara, CA 93106, USA ( $\{$ bamieh, khammash\}@engineering.ucsb.edu).

$\dagger$ This work is supported by research funding from the National Science Foundation through Grants ECCS-0835847 and ECCS-0802008 and funding from the NIH through grant R01-GM04983 and the Institute for Collaborative Biotechnologies through Grant DAAD19-03-D-0004 from the US Army Research Office.
}

In [2], two different techniques are applied to reason about the robustness of an oscillatory model. Another method to quantify the robustness of oscillatory behavior of biomolecular models to perturbations is presented in [3]. The authors propose a method that is based on Hopf bifurcation and Routh-Hurwitz stability criterion. In [4], a passivitybased stability criterion for a class of interconnected systems is discussed which extends the earlier work of the authors on the secant criterion for cyclic systems to a general interconnection structure [5]. The main result of [4] establishes global asymptotic stability of an interconnected network from the diagonal stability of the corresponding dissipativity matrix.

There is a large class of biochemical processes that can be represented using power-law expansions in the variables of the system. In this paper, we consider a special class of quasi-polynomial systems that arises in many biological applications. The state variables of the quasi-polynomial system represents one of the variables of the model (metabolite concentrations, protein concentrations or levels of gene expression) and the coefficients are stoichiometric coefficients and kinetic orders. The principal difference of quasipolynomial models with respect to other ODE models used in biochemical systems is that the kinetic orders can be noninteger numbers. A kinetic order can have even negative value when inhibition is modeled. In this way, powerlaw models have a higher flexibility to reproduce the nonlinearity of biochemical systems.

The objective of this paper is to analyze the stability properties of a given quasi-polynomial system by studying a mathematically equivalent counterpart of the system that has much simpler form to work with, namely, the LotkaVolterra system. We explain that a quasi-polynomial system can be transformed into a Lotka-Volterra system with some appropriate change of variables [6]. It is known that if the interaction matrix in Lotka-Volterra system ( $\Delta$ in (4)) is diagonally stable, then one can conclude the global asymptotic stability of the equilibrium of the system in the positive orthant [7], [8]. We may interpret the process of casting a quasi-polynomial system in the form of a Lotka-Volterra system as lifting the state-space to higher dimensions. The dimension of the corresponding LotkaVolterra system depends on the number of different quasimonomials appearing in the right-hand-side of the equations, which is usually greater than the number of state variables. Clearly, the resulting interaction matrix is singular. For a singular interaction matrix, the existing diagonal stability results can only guarantee the boundedness of the solutions in the positive orthant. Our contribution in this paper is to derive sufficient conditions under which the global 
asymptotic stability of the equilibria of the quasi-polynomial system in the positive orthant is guaranteed. These sufficient conditions impose a rank condition on the matrix of kinetic orders $(\Sigma$ in (1)) and require a comparison matrix constructed using the moduli of the entries of the interaction matrix to be an $M$-matrix. We also provide necessary conditions for asymptotic stability of the equilibria of the system. These results are applied to parameterized models of three different biological systems: generalized mass action (GMA) model, an oscillating biochemical network, and a reduced order model with Hill function. We show that one can find the range of parameters for which a given parameterized model is stable. The future work in this area will focus on developing multi-parametric optimization techniques to find regions in the parameter space for which the system is stable.

\section{NOTATIONS}

We denote the set of real numbers by $\mathbb{R}$. The positive orthant of $\mathbb{R}^{n}$ is defined as

$$
\mathbb{R}_{+}^{n}=\left\{x \in \mathbb{R}^{n} \mid x_{i}>0 \text { for all } i=1, \ldots, n\right\} .
$$

For a given matrix $A=\left[a_{i j}\right]$, we define matrix $M(A)=$ $\left[m_{i j}\right]$ as follows

$$
m_{i j}=\left\{\begin{array}{rll}
a_{i j} & \text { if } & j=i \\
\left|a_{i j}\right| & \text { if } & j \neq i
\end{array}\right.
$$

Definition 1: A matrix $A$ is called an $M$-matrix if $a_{i j} \leq 0$ for all $i \neq j$ and all the leading principle minors of $A$ are non-negative, or equivalently, if all the eigenvalues of $A$ have non-negative real parts.

\section{Global Stability of QUASI-Polynomial SySTEMS}

Most of the biochemical processes can be represented using power-law expansions in the variables of the system. In this paper, we consider the following class of quasipolynomial systems:

$$
\dot{x}_{i}=b_{i} x_{i}+x_{i} \sum_{j=1}^{m} a_{i j} \prod_{k=1}^{n} x_{k}^{\sigma_{j k}}
$$

for $i=1, \ldots, n$. The state variables $x_{i}$ represents one of the $n$ variables of the model (metabolite concentrations, protein concentrations or levels of gene expression), $b_{i}$ and $a_{i j}$ are stoichiometric coefficients, and $\sigma_{j k}$ are kinetic orders. The principal difference of quasi-polynomial models with respect to other ODE models used in biochemical systems is that the kinetic orders can be non-integer numbers. A kinetic order can have even negative value when inhibition is modeled. In this way, power-law models have a higher flexibility to reproduce the non-linearity of biochemical systems. We denote the trajectory of system (1) at time instant $t$ with initial condition $x(0)$ by $\psi_{t}(x(0))$.

Let define the interaction matrix as $A=\left[a_{i j}\right]$, the matrix of kinetic orders $\Sigma=\left[\sigma_{i j}\right]$, and $b=\left[b_{i}\right]$. We denote the set of all nontrivial equilibria of system (1) as $\mathcal{E}$ where

$$
\mathcal{E}=\left\{x^{*} \mid b_{i}+\sum_{j=1}^{m} a_{i j} \prod_{k=1}^{n}\left(x_{k}^{*}\right)^{\sigma_{j k}}=0, \quad i=1, \ldots, n\right\}
$$

We define the following change of variables

$$
z_{j}=\prod_{k=1}^{n} x_{k}^{\sigma_{j k}}
$$

This change of variables defines a lifting map $F: \mathbb{R}^{n} \rightarrow \mathbb{R}^{m}$. We denote the projection of the positive orthant $\mathbb{R}_{+}^{n}$ by $\Phi$ as

$$
\Omega=\left\{z \in \mathbb{R}_{+}^{m} \mid z=F(x) \text { for all } x \in \mathbb{R}_{+}^{n}\right\}
$$

The class of quasi-polynomial systems defined by (1) can be cast as a higher-dimensional Lotka-Volterra system with the following canonical form [6]

$$
\dot{z}_{i}=\lambda_{i} z_{i}+z_{i} \sum_{j=1}^{m} \delta_{i j} z_{j}
$$

for $i=1, \ldots, m$, where the system matrices are given by

$$
\Delta=\left[\delta_{i j}\right]=\Sigma A \quad \text { and } \quad \lambda=\left[\lambda_{i}\right]=\Sigma b .
$$

Although there is little, apparently, in common between models (4) and (1), these two systems are mathematically equivalent and do share the same behavior if $z(0) \in \Omega$. We denote the trajectory of system (4) at time instant $t$ with initial condition $z(0)$ by $\phi_{t}(z(0))$.

The stability properties of (4) was studied in [7]. For a recent reference on the subject, we refer to [8] for more detailed. The following theorem from [7] gives a sufficient condition for the global stability of system (4).

Theorem 1: If there exists a constant positive diagonal matrix $P=\operatorname{diag}\left(p_{1}, \ldots, p_{m}\right)>0$ such that

$$
\Delta^{T} P+P \Delta<0
$$

then the nontrivial equilibrium $z^{*} \in \mathbb{R}_{+}^{m}$ of the LotkaVolterra model (4) is globally stable for all $z(0) \in \mathbb{R}_{+}^{m}$.

Proof: We refer to [7] for a proof.

The existence of a positive diagonal matrix in Theorem 1 implies that $\Delta$ is non-singular and that asymptotic stability of the unique nontrivial equilibrium. In order to handel nonsingular $\Delta$, the sufficient condition in Theorem 1 can be relaxed to the following form

$$
\Delta^{T} P+P \Delta \leq 0
$$

for a positive diagonal matrix $P$. The existence of a solution for (6) implies the boundedness of the solutions and stability of the nontrivial equilibrium points. It is straightforward to verify that the following function which is defined on $\mathbb{R}_{+}^{m}$ serves as a Lyapunov candidate for system (4)

$$
V(z)=\sum_{i=1}^{m} p_{i}\left(z_{i}-z_{i}^{*}-z_{i}^{*} \ln \left(\frac{z_{i}}{z_{i}^{*}}\right)\right)
$$

in which $z^{*}$ is a nontrivial equilibrium of (4) and $P=$ $\operatorname{diag}\left(p_{1}, \ldots, p_{m}\right)>0$ satisfies (5) or (6). We refer to [8] for 
a thorough discussion on diagonal stability and the related diagonal-type Lyapunov functions.

Let assume that the number of monomial $m$ is greater than the number of state variables $n$ in (1). Thus, one can see that $\Delta=\Sigma A$ is a singular matrix. By applying Theorem 1 to system (4), we can only hope to prove the boundedness of the solutions of (4), and therefore, the solutions of (1). In the following theorem, we propose sufficient conditions for asymptotic stability of the set of the equilibrium points of (1).

Theorem 2: Suppose that $\mathcal{E}$ is the set of all equilibria of system (1) in $\mathbb{R}_{+}^{n}$ and matrix $\Delta=\Sigma A$ is irreducible. Then every trajectory of the system $\psi_{t}(x(0))$ asymptotically converges to the set $\mathcal{E}$ for all initial conditions $x(0) \in \mathbb{R}_{+}^{n}$ if $\operatorname{rank}(\Sigma)=n$ and $-M(\Delta)$ is an $M$-matrix.

Proof: Let consider function (7) where $z^{*}=F\left(x^{*}\right)$ for some $x^{*} \in \mathcal{E}$. We show that one can choose parameters $p_{i}>0$ such that the time derivative of (7) is non-positive along all trajectories $\phi_{t}(z(0))$ of system (4) with $z(0) \in \Omega$. The time-derivative of (7) along a trajectory of (4) is given by

$$
\dot{V}=\sum_{i=1}^{m} p_{i} \dot{z}_{i}\left(\frac{z-z_{i}^{*}}{z_{i}}\right) .
$$

We can rewrite (4) in terms of new state variables $y_{i}=z_{i}-z_{i}^{*}$ as follows

$$
\dot{z}_{i}=z_{i} \sum_{j=1}^{m} \delta_{i j} y_{j}
$$

By plugging this into (8) we get

$$
\begin{aligned}
\dot{V} & =\sum_{i=1}^{m} p_{i} y_{i} \sum_{j=1}^{m} \delta_{i j} y_{j} \\
& =\sum_{i=1}^{m} \sum_{j=1}^{m} p_{i} \delta_{i j} y_{i} y_{j}+\sum_{i=1}^{m} q_{i} y_{i}^{2}-\sum_{i=1}^{m} q_{i} y_{i}^{2}
\end{aligned}
$$

In the later equation, the last two terms add up to zero, but they will help us to prove asymptotic stability of the equilibrium. The assumption that $-M(\Delta)$ is an $M$-matrix (and irreducible) is equivalent to the fact that there exist vectors $\mu, \nu$ such that (for example, see Theorem 5.9 in [9])

$$
M(\Delta) \nu \leq 0, \quad \nu>0 \quad \text { and } \quad \mu^{T} M(\Delta) \leq 0, \quad \mu>0 .
$$

In other words, there exist $\nu_{i}, \mu_{i}>0$ such that

$$
\begin{aligned}
& \delta_{i i} \nu_{i}+\sum_{j \neq i}\left|\delta_{i j}\right| \nu_{j} \leq 0 \\
& \mu_{i} \delta_{i i}+\sum_{j \neq i} \mu_{j}\left|\delta_{j i}\right| \leq 0
\end{aligned}
$$

By choosing $p_{i}=\frac{2 \mu_{i}}{\nu_{i}}$ and $q_{i}=\frac{2}{\nu_{i}} \sum_{j \neq i} \mu_{j}\left|\delta_{j i}\right|$, we have

$$
\begin{aligned}
\dot{V}=2 \sum_{i=1}^{m} \sum_{j \neq i}^{m} \frac{\mu_{i}}{\nu_{i}} \delta_{i j} y_{i} y_{j} & \\
+\sum_{i=1}^{m}\left(\sum_{j \neq i} \frac{\mu_{j}}{\nu_{i}}\left|\delta_{j i}\right|\right. & \left.+2 \frac{\mu_{i}}{\nu_{i}} \delta_{i i}\right) y_{i}^{2} \\
& -\sum_{i=1}^{m} \sum_{j \neq i} \frac{\mu_{j}}{\nu_{i}}\left|\delta_{j i}\right| y_{i}^{2}
\end{aligned}
$$

From (11) and (12), we have that

$$
\frac{\mu_{i}}{\nu_{i}^{2}}\left(\delta_{i i} \nu_{i}+\sum_{j \neq i}\left|\delta_{i j}\right| \nu_{j}\right)+\frac{1}{\nu_{i}}\left(\mu_{i} \delta_{i i}+\sum_{j \neq i} \mu_{j}\left|\delta_{j i}\right|\right) \leq 0
$$

Therefore, it follows that

$$
\sum_{j \neq i} \frac{\mu_{j}}{\nu_{i}}\left|\delta_{j i}\right|+2 \frac{\mu_{i}}{\nu_{i}} \delta_{i i} \leq-\sum_{j \neq i} \frac{\mu_{i}}{\nu_{i}^{2}} \nu_{j}\left|\delta_{i j}\right|
$$

By applying (14) to (13), we get the following inequality

$$
\dot{V} \leq-\sum_{j \neq i} \frac{\mu_{i}}{\nu_{j}}\left|\delta_{i j}\right|\left(\frac{\nu_{j}}{\nu_{i}} \operatorname{sgn}\left(\delta_{i j}\right) y_{i}-y_{j}\right)^{2} \leq 0
$$

The boundedness of $V$ for a positive solution $z(t)$ implies the boundedness of the state variables, i.e., $m \leq z_{i}(t) \leq M$ for some $m, M>0$ and all $t \geq 0$. According to LaSalle's theorem, the $\omega$-limit set of the system is contained in the maximal invariant subset of $\left\{z \in \mathbb{R}^{n} \mid \dot{V}(z) \equiv 0\right\}$.

From (15), one can see that if $\dot{V} \equiv 0$, then $y_{j}=$ $\frac{\nu_{j}}{\nu_{i}} \operatorname{sgn}\left(\delta_{i j}\right) y_{i}$. By substituting this into (9), we get

$$
\dot{V}=\sum_{i=1}^{m}\left(\frac{2 \mu_{i}}{\nu_{i}^{2}}\right)\left(\delta_{i i} \nu_{i}+\sum_{j \neq i}\left|\delta_{i j}\right| \nu_{j}\right) y_{i}^{2} \equiv 0 .
$$

Let suppose that $z_{i}(t) \not \equiv z_{i}^{*}$ whenever $\dot{V} \equiv 0$. From inequalities (11) and (12), we can conclude that $\dot{V} \equiv 0$ if and only if $\delta_{i i} \nu_{i}+\sum_{j \neq i}\left|\delta_{i j}\right| \nu_{j}=0$. This implies that

$$
\dot{z}_{i}=z_{i}\left(\delta_{i i} \nu_{i}+\sum_{j \neq i}\left|\delta_{i j}\right| \nu_{j}\right) \frac{y_{i}}{\nu_{i}}=0
$$

Hence $z(t)$ must be a constant solution of (4). Using our assumption that $z(0) \in \Omega$, we can characterize the set of such constant solutions as follows

$$
\left\{z \in \mathbb{R}_{+}^{m} \mid \lambda+\Delta z=0\right\} \cap \Omega .
$$

Since $\operatorname{rank}(\Sigma)=n$, the set of solutions reduces to $\left\{z \in \mathbb{R}_{+}^{m} \mid b+A z=0\right\} \cap \Omega=\left\{z^{*} \mid z^{*}=F\left(x^{*}\right), \forall x^{*} \in \mathcal{E}\right\}$.

Therefore, the maximal invariant set of system (4) only contains the set of equilibria $\mathcal{E}$. This implies that $\phi_{t}(x(0))$ for all $x(0) \in \mathbb{R}_{+}^{n}$ converges asymptotically to $\mathcal{E}$.

Corollary 1: For system (1), assume that $\Delta$ is diagonally dominant. If all the trajectories $\psi_{t}(x(0))$ of (1) asymptotically converges to the set of equilibria $\mathcal{E}$ for all initial conditions $x(0) \in \mathbb{R}_{+}^{n}$, then $\operatorname{rank}(\Sigma)=n$ and $-M(\Delta)$ is 
an $M$-matrix.

Proof: We consider system (4) with $z(0) \in \Omega$. Thus, we only need to consider the set of all possible equilibria of (4) on $\Omega$, i.e.,

$$
\left\{z \in \mathbb{R}_{+}^{m} \mid \Sigma(b+A z)=0\right\} \cap \Omega .
$$

Then, $\operatorname{rank}(\Sigma)=n$, because otherwise (4) has equilibrium solutions other than those in $\left\{z^{*} \mid z^{*}=F\left(x^{*}\right), \forall x^{*} \in \mathcal{E}\right\}$ and this implies that $\mathcal{E}$ is not asymptotically stable. Now, let consider the linearization of (4) at an equilibrium point $z^{*}$ given by

$$
J=\operatorname{diag}\left(z_{1}^{*}, \ldots, z_{m}^{*}\right) \Delta .
$$

Since $\mathcal{E}$ is asymptotically stable, all the eigenvalues of $J$ must have non-positive real parts. Therefore, all the eigenvalues of $\Delta$ must have non-positive real parts. From our assumption that $\Delta$ is diagonally dominant and according to Gershgorin circle theorem, the eigenvalues of $M(\Delta)$ also belong to the Gershgorin discs of matrix $\Delta$. Therefore, all the eigenvalues $-M(\Delta)$ must have non-negative real parts. This implies that $-M(\Delta)$ must be an $M$-matrix.

Theorem 2 characterizes sufficient conditions for the stability of the set of equilibria of system (1). The condition $-M(\Delta)$ is an $M$-matrix is equivalent to the following feasibility condition: there is a positive diagonal matrix $D$ such that

$$
D M(\Delta)+M(\Delta)^{T} D<0 .
$$

The sufficient condition provided by Theorem (2) is more conservative than that of Theorem (1). However, it guarantees asymptotic stability of the equilibria. We refer the reader to [8] for an extensive discussion on how to check the feasibility and solve linear matrix inequality (16).

\section{Case Studies}

\section{A. Generalized Mass Action (GMA) Model}

In this example, we consider stability conditions for the following Generalized Mass Action model for biochemical reactions [10]

$$
\begin{aligned}
& \dot{x}_{1}=b_{1} x_{1}-a_{1} x_{1}^{\sigma_{3}} x_{2}^{\sigma_{1}} \\
& \dot{x}_{2}=-b_{2} x_{2}+a_{2} x_{1}^{\sigma_{1}} x_{3}^{\sigma_{2}} \\
& \dot{x}_{3}=-b_{3} x_{3}+a_{3} x_{2}^{\sigma_{1}}
\end{aligned}
$$

The state variables $x_{i}>0$ are concentrations, parameters $\sigma_{i}$ are rates of different processes, and $b_{i}, a_{i}>0$ are the reaction rates. This system can be cast as (1) with the following matrices

$$
b=\left[\begin{array}{c}
b_{1} \\
-b_{2} \\
-b_{3}
\end{array}\right], A=\left[\begin{array}{ccc}
-a_{1} & 0 & 0 \\
0 & a_{2} & 0 \\
0 & 0 & a_{3}
\end{array}\right], \Sigma=\left[\begin{array}{ccc}
\sigma_{3}-1 & \sigma_{1} & 0 \\
\sigma_{1} & -1 & \sigma_{2} \\
0 & \sigma_{1} & -1
\end{array}\right]
$$

For instance, the dynamical system (17)-(19) models an immune network with suppressor regulation [10] for the following parameters

$b=\left[\begin{array}{c}8 \\ -1 \\ -1\end{array}\right], A=\left[\begin{array}{ccc}-8 & 0 & 0 \\ 0 & 1 & 0 \\ 0 & 0 & 1\end{array}\right], \Sigma=\left[\begin{array}{ccc}0 & \frac{1}{2} & 0 \\ \frac{1}{2} & -1 & -\frac{1}{2} \\ 0 & \frac{1}{2} & -1\end{array}\right]$
It is straightforward to verify that the equilibrium of the corresponding Lotka-Volterra system is given by

$$
z_{1}^{*}=\frac{b_{1}}{a_{1}}, z_{2}^{*}=\frac{b_{2}}{a_{2}}, z_{3}^{*}=\frac{b_{3}}{a_{3}} .
$$

According to Theorem 2, necessary and sufficient conditions for stability of system (17)-(19) are $\operatorname{rank}(\Sigma)=3$ and

$$
\left[\begin{array}{ccc}
\left(\sigma_{3}-1\right) a_{1} & -\left|\sigma_{1}\right|\left|a_{2}\right| & 0 \\
-\left|\sigma_{1}\right|\left|a_{1}\right| & a_{2} & -\left|\sigma_{2}\right|\left|a_{3}\right| \\
0 & -\left|\sigma_{1}\right|\left|a_{2}\right| & a_{3}
\end{array}\right] \text { is an } M \text {-matrix. }
$$

Thus, one can easily compute all the principle minors of the above matrix and find the set of parameters for which system (17)-(19) is globally asymptotically stable in the positive orthant.

\section{B. Oscillating Biochemical Network}

In our next example, we consider a model of the molecular network underlying adenosine 3',5'-cyclic monophosphate (cAMP) oscillations observed in homogenous populations of Dictyostelium cells [11]. The proposed model exhibits the spontaneous oscillations in cAMP observed during the early development of Dictyostelium discoideum. The robustness properties of this model was studied in [2], [3].

The variations in the enzymatic activities of these proteins are described by the following autonomous dynamical system

$$
\left\{\begin{array}{c}
\dot{x}_{1}=k_{1} x_{7}-k_{2} x_{1} x_{2} \\
\dot{x}_{2}=k_{3} x_{5}-k_{4} x_{2} \\
\dot{x}_{3}=k_{5} x_{7}-k_{6} x_{2} x_{3} \\
\dot{x}_{4}=k_{7}-k_{8} x_{3} x_{4} \\
\dot{x}_{5}=k_{9} x_{1}-k_{10} x_{4} x_{5} \\
\dot{x}_{6}=k_{11} x_{1}-k_{12} x_{6} \\
\dot{x}_{7}=k_{13} x_{6}-k_{14} x_{7}
\end{array}\right.
$$

The unique equilibrium of the system in $\mathbb{R}_{+}^{7}$ in terms of parameters $k_{i}$ can be calculated analytically (see [3] for more details). It is straightforward to see that one can reformulate (20) in the form of (1) with the following quasi-monomials $x_{2}, x_{3}, x_{4}, x_{4}^{-1}, x_{1}^{-1} x_{7}, x_{2}^{-1} x_{5}, x_{3}^{-1} x_{7}, x_{1} x_{4}^{-1}, x_{1} x_{6}^{-1}, x_{6} x_{7}^{-1}$ and matrices

$$
\begin{gathered}
b=\left[\begin{array}{c}
0 \\
-k_{4} \\
0 \\
0 \\
0 \\
-k_{12} \\
-k_{14}
\end{array}\right] \\
A=\left[\begin{array}{cccccccccc}
-k_{2} & 0 & 0 & 0 & k_{1} & 0 & 0 & 0 & 0 & 0 \\
0 & 0 & 0 & 0 & 0 & k_{3} & 0 & 0 & 0 & 0 \\
-k_{6} & 0 & 0 & 0 & 0 & 0 & k_{5} & 0 & 0 & 0 \\
0 & -k_{8} & 0 & k_{7} & 0 & 0 & 0 & 0 & 0 & 0 \\
0 & 0 & -k_{10} & 0 & 0 & 0 & 0 & k_{9} & 0 & 0 \\
0 & 0 & 0 & 0 & 0 & 0 & 0 & 0 & k_{11} & 0 \\
0 & 0 & 0 & 0 & 0 & 0 & 0 & 0 & 0 & k_{13}
\end{array}\right] \\
\Sigma= \\
\quad\left[\begin{array}{ccccccccc}
0 & 1 & 0 & 0 & 0 & 0 & 0 \\
0 & 0 & 1 & 0 & 0 & 0 & 0 \\
0 & 0 & 0 & 1 & 0 & 0 & 0 \\
0 & 0 & 0 & -1 & 0 & 0 & 0 \\
-1 & 0 & 0 & 0 & 0 & 0 & 1 \\
0 & -1 & 0 & 0 & 1 & 0 & 0 \\
0 & 0 & -1 & 0 & 0 & 0 & 1 \\
1 & 0 & 0 & -1 & 0 & 0 & 0 \\
1 & 0 & 0 & 0 & 0 & -1 & 0 \\
0 & 0 & 0 & 0 & 0 & 1 & -1
\end{array}\right]
\end{gathered}
$$

The matrix $\Sigma$ has full-column rank. Also, the matrix $-M(\Delta)$ 


$$
\Delta=\left[\begin{array}{ccccccc}
k_{1} & -(q+1) k_{2} & q V & 0 & 0 & 0 & 0 \\
k_{1} & -(q+1) k_{2} & q V & V & 0 & 0 & 0 \\
-k_{1} q+k_{1} & k_{2} q^{2}-k_{2} & -q^{2} V+q V & 0 & \gamma q h V & -\gamma h(q+1) k_{2} & \gamma h k_{1} \\
-k_{1} q & k_{2} q^{2}+k_{2} q & -q^{2} V & -V & \gamma q h V & -\gamma h(q+1) k_{2} & \gamma h k_{1} \\
-k_{1} q-k_{1} h+k_{1} & k_{2} q^{2}+k_{2} h q+k_{2} h-k_{2} & -q^{2} V-q V h+q V & 0 & 2 \gamma q h V & -2 \gamma h(q+1) k_{2} & 2 \gamma h k_{1} \\
0 & 0 & 0 & V & \gamma q h V & -\gamma h(q+1) k_{2} & \gamma h k_{1} \\
-k_{1} h+k_{1} & k_{2} h q+k_{2} h-k_{2} q-k_{2} & -q V h+q V & 0 & \gamma q h V & -\gamma h(q+1) k_{2} & \gamma h k_{1}
\end{array}\right]
$$

Fig. 1. The $\Delta$ matrix of the nominal regulated autocatalytic glycolysis model

in which $\Delta=\Sigma A$ is given by

$$
-M(\Delta)=\left[\begin{array}{cccccccccc}
0 & 0 & 0 & 0 & 0 & -k_{3} & 0 & 0 & 0 & 0 \\
-k_{6} & 0 & 0 & 0 & 0 & 0 & -k_{5} & 0 & 0 & 0 \\
0 & -k_{8} & 0 & -k_{7} & 0 & 0 & 0 & 0 & 0 & 0 \\
0 & -k_{8} & 0 & k_{7} & 0 & 0 & 0 & 0 & 0 & 0 \\
-k_{2} & 0 & 0 & 0 & k_{1} & 0 & 0 & 0 & 0 & -k_{13} \\
0 & 0 & -k_{10} & 0 & 0 & k_{3} & 0 & -k_{9} & 0 & 0 \\
-k_{6} & 0 & 0 & 0 & 0 & 0 & k_{5} & 0 & 0 & -k_{13} \\
-k_{2} & -k_{8} & 0 & -k_{7} & -k_{1} & 0 & 0 & 0 & 0 & 0 \\
-k_{2} & 0 & 0 & 0 & -k_{1} & 0 & 0 & 0 & k_{11} & 0 \\
0 & 0 & 0 & 0 & 0 & 0 & 0 & 0 & -k_{11} & k_{13}
\end{array}\right]
$$

Thus, for all values of parameters $k_{i}$ for which $-M(\Delta)$ is an $M$-matrix, the equilibrium of the system (20) is globally asymptotically stable for all initial conditions in $\mathbb{R}_{+}^{7}$.

\section{Reduced Biological Models}

An important special class of biochemical models is that in which the Hill functions appear

$$
\frac{a x^{\alpha}}{1+b x^{\beta}}
$$

for some positive real numbers $a, b, \alpha, \beta$. For differential systems arising from generalized chemical reactions systems, there exists a standard way to perform the quasi-steady state approximation, provided that the set of chemical reactions is divided in two parts: the fast ones and the slow ones. One can obtain a set of algebraic equations by ignoring the dynamics of the fast dynamics (by setting the time-derivative of the fast dynamics equal to zero). There is an standard procedure by which one can obtain a reduced model which only contains the slow dynamics. These reduced models usually contain the Hill functions. In this example, we consider the nominal regulated autocatalytic glycolysis model, which is studied in [1], as follows

$$
\begin{aligned}
\dot{x} & =-q \frac{V x^{q}}{1+\gamma x^{h}}+(1+q) k_{2} y-k_{1} \\
\dot{y} & =\frac{V x^{q}}{1+\gamma x^{h}}-k_{2} y
\end{aligned}
$$

in which $x$ is the ATP level, $y$ the lumped variable of intermediate metabolites downstream of the autocatalytic reaction, $q$ captures the strength of autocatalysis, $k_{2}$ represents the lumped metabolic reactions that generate ATP, $k_{1}$ represents the ATP demand of the cell, and $h$ is the gain of the inhibition of the enzymes by ATP. The parameter $\gamma$ is determined by the enzyme and regulate the strength of feedback inhibition. $V$ is related to the availability of precursors such as F6P.

In the following, we show that by a suitable change of variable, one can cast a nonlinear system with Hill functions in the form of (1). For example, let consider the auxiliary variable defined by

$$
z=\frac{x y}{1+\gamma x^{h}} .
$$

This new variable does not have a biological interpretation. However, it helps us to reformulated the glycolysis model in the following quasi-polynomial representation

$$
\begin{aligned}
\dot{x}= & x\left(-q V x^{q-2} y^{-1} z+(q+1) k_{2} x^{-1} y-k_{1} x^{-1}\right) \\
\dot{y}=y & \left(V x^{q-1} y^{-2} z-k_{2}\right) \\
\dot{z}=z & \left(-q V x^{q-2} y^{-1} z+(q+1) k_{2} x^{-1} y-k_{1} x^{-1}\right. \\
& +V x^{q-1} y^{-2} z-k_{2}+\gamma q h V x^{q+h-3} y^{-2} z^{2} \\
& \left.-\gamma h(q+1) k_{2} x^{-1} z+\gamma h k_{1} x^{h-2} y^{-1} z\right)
\end{aligned}
$$

Now, we can extract the corresponding matrices in the canonical representation of the system from (23) as follows

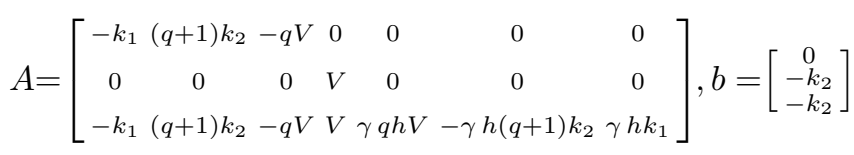

and

$$
\Sigma=\left[\begin{array}{ccc}
-1 & 0 & 0 \\
-1 & 1 & 0 \\
q-2 & -1 & 1 \\
q-1 & -2 & 1 \\
q+h-3 & -2 & 2 \\
-1 & 0 & 1 \\
h-2 & -1 & 1
\end{array}\right]
$$

The $\Delta$ matrix of the system is shown in Fig. 1. It is straightforward to verify that $\operatorname{rank}(\Sigma)=3$. Therefore, a sufficient condition for the positive equilibrium of the system (21)-(22) to be globally asymptotically stable is that $-M(\Delta)$ to be an $M$-matrix. we should emphasize that the representation (23) is not unique. Therefore, one may able be to find a suitable equivalent quasi-polynomial representation of (21)-(22) that can provide more insight into the stability properties of the glycolysis model. 


\section{CONCLUSION}

We study the stability conditions for a special class of quasi-polynomial system arisen in several biochemical applications.We show that a given quasi-polynomial systems can be cast as a higher dimensional singular Lotka-Volterra system. We proved that under some sufficient conditions the solutions of the quasi-polynomial systems converge to the set of equilibrium points. We apply our results to three different biological models: generalized mass action (GMA) model, an oscillating biochemical network, and a reduced order model with Hill function. We show that one can find the range of parameters for which a given parameterized model is stable.

\section{REFERENCES}

[1] F. A. Chandra, G. Buzi, and J. C. Doyle, "Linear control analysis of the autocatalytic glycolysis system," in Proc. of the American Control Conference, 2009.

[2] L. Ma and P. A. Iglesias, "Quantifying robustness of biochemical network models," BMC Bioinformatics, vol. 3:38, pp. 38-50.

[3] R. Ghaemi, J. Sun, P. Iglesias, and D. D. Vecchio, "A method for determining the robustness of bio-molecular oscillator models," $B M C$ Systems Biology, vol. To appear.

[4] M. Arcak and E. Sontag, "A passivity-based stability criterion for a class of biochemical reaction networks," Mathematical biosciences and engineering, vol. 5, no. 1, pp. 1-19, 2008.

[5] — "Diagonal stability of a class of cyclic systems and its connection with the secant criterion," Automatica, vol. 42, no. 9, p. 15311537.

[6] B. Hernndez-Bermejoa and V. Fairen, "Lotka-volterra representation of general nonlinear systems," Mathematical Biosciences, vol. 140, no. 1, pp. 1-32, 1997.

[7] B. S. Goh, "Global stability in many-species systems," The American Naturalist, vol. 111, no. 977, pp. 135-143, 1977.

[8] E. Kaszkurewicz and A. Bhaya, Matrix Diagonal Stability in Systems and Computation. Birkhauser, Boston, 2000.

[9] M. Fiedler, Special Matrices and Their Applications in Numerical Mathematics. Martinus Nijhoff Publications (Kluwer), Dordrecht, 1986.

[10] D. Irving, E. Voit, and M. Savageau, Analysis of Complex Dynamic Networks with ESSYNS. In E.O. Voit Ed., Canonical Non-Linear Modelling S-systems Approach to Understanding Complexity, Von Nostrand, Reinhold, 1991.

[11] M. Laub and W.F.Loomis, "A molecular network that produces spontaneous oscillations in excitable cells of dictyostelium," Molecular Biology of the Cell, vol. 9, pp. 3521-3532, 1998. 